\title{
Correlation of neuter status and expression of heritable disorders
}

\author{
Janelle M. Belanger ${ }^{1}$, Thomas P. Bellumori', Danika L. Bannasch, Thomas R. Famula ${ }^{1}$ and Anita M. Oberbauer ${ }^{1 *}$
}

\begin{abstract}
Background: Gonadectomy, or neutering, is a very common surgery for dogs having many positive effects on behavior, health, and longevity. There are also certain risks associated with neutering including the development of orthopedic conditions, cognitive decline, and a predisposition to some neoplasias. This study was designed specifically to identify if a correlation exists between neuter status and inherited conditions in a large aggregate cohort of dogs representing many different breeds.

Results: Neutered dogs were at less risk for early and congenital conditions (aortic stenosis, early onset cataracts, mitral valve disease, patent ductus arteriosus, portosystemic shunt, and ventricular septal defect) than intact dogs. Neutering was also associated with reduced risk of dilated cardiomyopathy and gastric dilatation volvulus in males. Neutering was significantly associated with an increased risk for males and females for cancers (hemangiosarcoma, hyperadrenocorticism, lymphoma, mast cell tumor, and osteosarcoma), ruptured anterior cruciate ligament and epilepsy. Intervertebral disk disease was associated with increased risk in females only. For elbow dysplasia, hip dysplasia, lens luxation, and patellar luxation neutering had no significant effect on the risk for those conditions. Neutering was associated with a reduced risk of vehicular injury, a condition chosen as a control.

Conclusions: In this retrospective study, several conditions showed an increased risk associated with neutering whereas other conditions were less likely to be expressed in neutered dogs. The complexity of the interactions between neutering and inherited conditions underscores the need for reflective consultation between the client and the clinician when considering neutering. The convenience and advantages of neutering dogs that will not be included in a breeding program must be weighed against possible risk associated with neutering.
\end{abstract}

Keywords: Neuter, Dog, Inherited disease

\section{Plain English summary}

Spaying and neutering of dogs is a well-accepted procedure in the United States and has many positive effects on behavior, health, and longevity. Although recent reports suggest that spaying and neutering may increase the occurrence of some joint disorders and some cancers, the relationship between inherited diseases and spay/neuter status has not fully been explored. The present study evaluated the prevalence and risk of genetic diseases, both early and late onset, in intact and neutered male and female dogs that were seen over a 15 -year period at a university teaching hospital. Spayed and neutered dogs were at less risk for early and

\footnotetext{
* Correspondence: amoberbauer@ucdavis.edu

${ }^{1}$ Department of Animal Science, University of California, One Shields Ave,

Davis, CA 95616, USA

Full list of author information is available at the end of the article
}

congenital conditions (aortic stenosis, early onset cataracts, mitral valve disease, patent ductus arteriosus, portosystemic shunt, and ventricular septal defect) than intact dogs. Neutered male dogs were at less risk for bloat (gastric dilatation volvulus) and dilated cardiomyopathy, whereas spayed females were at increased risk for intervertebral disk disease. Spaying or neutering in both sexes was significantly associated with an increased risk for cancers (hemangiosarcoma, hyperadrenocorticism, lymphoma, mast cell tumor, and osteosarcoma), ruptured anterior cruciate ligament, and epilepsy. For elbow dysplasia, hip dysplasia, lens luxation, and patellar luxation neutering had no significant effect on the risk for those conditions. A dog that was spayed or neutered was associated with a reduced risk of vehicular injury, a condition chosen as a control. The complexity of the interactions between spaying/neutering and inherited 
conditions underscores the need for reflective consultation between the client and the clinician when considering the procedure. The convenience and advantages of spaying or neutering dogs that will not be included in a breeding program must be weighed against possible risk associated with the procedure. Additionally, if owners elect to keep their dogs intact, they must then assume responsibility to vigilantly guard against unplanned litters.

\section{Background}

Gonadectomy, or neutering, is a very common elective surgery in dogs. Current reports from the ASPCA of the United States indicate $83 \%$ of male and female dogs are neutered (http://www.aspca.org/animal-homelessness/ shelter-intake-and-surrender/pet-statistics). Neutering has a number of benefits including eliminating unintended reproduction which in turn reduces the number of dogs that are unwanted and euthanized in shelters. Reproduction, pregnancy, and parturition are also associated with adverse health conditions such as sexually transmitted disease, pregnancy toxemia, metabolic disease, and dystocia [1, 2]. Reproductive disorders such as pyometra, mammary tumors, testicular cancer, ovarian cancer, and prostate cancer are also reduced/prevented with neutering (reviewed in [2]) although neutering has been associated with increased risk or aggressiveness of prostatic cancer [3-5]. Numerous studies evaluating the correlation of behavior with neutering have shown reduced aggression, mounting behavior, roaming, and urine marking $[6,7]$. Neutering reduces the risk of biting in certain breeds of dogs $[8,9]$ and dogs that are neutered are at reduced risk for relinquishment [10]. Additionally, neutered dogs have been shown to have increased lifespan as compared to intact animals by 13.8 and $26.3 \%$ for males and females, respectively [11].

Studies have also shown that there are certain risks associated with neutering. Certain orthopedic conditions appear to be increased in neutered dogs [12-16]. Cognitive decline of aging dogs is accelerated in the neutered state [17] and neutering has been correlated with greater prevalence of immune disorders [18]. Despite the reduction in mammary and gonadal neoplasia, neutering is associated with increased prevalence of other cancers such as hemangiosarcoma, lymphosarcoma, mast cell tumor, and osteosarcoma [12, 13, 19-21].

The goal of this study was to assess if there exists a correlation between neuter status and diseases having a presumed inherited basis by evaluating a large cohort of dogs representing many different breeds. We hypothesized that for certain conditions, removing gonadal hormones by neutering would reduce the risk of disease expression whereas for other conditions, neutering would be associated with an increased risk of expression. Appreciating the limitations of a retrospective study and that not all dog breeds would exhibit all the conditions under study, the cohort of dogs seen at a teaching hospital were analyzed in aggregate with breed analysis secondary.

\section{Methods}

Medical records for 90,090 individual dogs seen at the University of California William T. Pritchard Teaching Hospital from 1995 through the end of 2010 (a 15 year period) were categorized using methodology as previously described [22]. Dogs were classified as having one of the disease conditions if a confirmed diagnosis based on symptoms and test results was indicated in the records; diagnoses described as "possible" or "suspect" were not classified as a case nor were they considered unaffected (Additional file 1: Table S1). The entire medical record for each dog seen was interrogated for the diseases evaluated. Breed, age, and whether the dog was intact, or neutered was also tabulated. The date of initial diagnosis, if possible to determine from the medical record, was recorded; dogs with known neutering dates within 150 days of an initial diagnosis were classified as intact at the time of diagnosis. For the majority of dogs, age of neuter was not discernible from the records thereby precluding an assessment of age of neuter effects.

The disease conditions assessed were chosen because they are considered to be inherited (Online Mendelian Inheritance in Animals (OMIA) website (http://omia.angis. org.au/home/ and [23, 24]), have a relative surety of diagnosis, impact quality of life, and present in a high enough prevalence to permit analyses. Some of the conditions can also be considered congenital and those were: aortic stenosis, mitral valve disease, patent ductus arteriosus (PDA), portosystemic shunt, and ventricular septal defect [25-27]. The ocular disorders were lens luxation and early onset cataracts (those that appear at age 6 or earlier) [28] and orthopedic disorders were elbow dysplasia, hip dysplasia, intervertebral disk disease (IVDD), patellar luxation, and ruptured anterior cruciate ligament (RACL). Cancers evaluated were hemangiosarcoma, lymphoma, mast cell tumor, and osteosarcoma [23]. Hyperadrenocorticism, which can be the result of either hyperplastic overgrowth of pituitary adrenocorticotropin cells or tumors of the adrenal cortex [29], was also evaluated. Other disorders included dilated cardiomyopathy, gastric dilatation volvulus (GDV), and epilepsy. Vehicular injuries were used as a non-inherited control condition. Age of reported diagnosis for intact and neutered males and females in each condition were compared using a Student's $T$-test with significance set at $P<0.05$.

Data were categorized as binary traits (case or control) for each condition. Overall prevalence was calculated for each condition and the conditions were further categorized into one of 157 breed groups as well as one of four sex classes: intact females (F), neutered females (NF), 
intact males (M), and neutered males (NM). The final set of breed groups encompassed 153 recognized by the American Kennel Club (AKC) and 4 groups representing various crossbreds, Pit bulls, the AKC Foundation Stock Service (FSS) breeds, and the AKC miscellaneous breeds. Using a binomial density, the differences in the probability of a condition by sex class and breed group was calculated. For a given condition with sex class $i$ (where $i=\mathrm{F}, \mathrm{NF}, \mathrm{M}$, $\mathrm{NM})$ and breed group $j(j=1,2,3, \ldots, 157)$ we assume that $\mathrm{n}_{\text {cases ij }} \sim$ Binomial $\left(\mathrm{n}_{\text {cases }} \mathrm{ij}+\mathrm{n}_{\text {controls }} \mathrm{ij}, \mathrm{p}_{\mathrm{ij}}\right)$ where $\mathrm{n}_{\text {cases ij }}$ was the number of observed cases in sex group $i$ and breed group $j$ and $\mathrm{n}_{\text {controls ij }}$ was the number of unaffected dogs in sex group $i$ and breed group $j$ and where $\mathrm{p}_{\mathrm{ij}}$ was the probability of disease in sex and breed group $i j$. For each condition, $\mathrm{p}_{\mathrm{ij}}$ for all $i$ and $j$, was estimated as well as the odds ratio (OR) across the two neuter states for females and for males [30]. That is, the odds ratios for females and males, pooled across breeds, were defined as $\mathrm{OR}_{\mathrm{F}}=\left[\mathrm{p}_{\mathrm{NF}} /\left(1-\mathrm{p}_{\mathrm{NF}}\right)\right] /\left[\mathrm{p}_{\mathrm{F}} /\left(1-\mathrm{p}_{\mathrm{F}}\right)\right]$ and $\mathrm{OR}_{\mathrm{M}}=\left[\mathrm{p}_{\mathrm{NM}} /(1-\right.$ $\left.\left.\mathrm{p}_{\mathrm{NM}}\right)\right] /\left[\mathrm{p}_{\mathrm{M}} /\left(1-\mathrm{p}_{\mathrm{M}}\right)\right]$, respectively.

Evaluating these probabilities and odds ratios was facilitated through a hierarchical Bayesian framework implemented through the public-domain software Stan [31], accessed through the public-domain language $R$ [32]. Using a hierarchical model permitted stable estimates of $\mathrm{p}_{\mathrm{ij}}$ and valid error intervals for $\mathrm{p}_{\mathrm{ij}}$ and therefore more credible OR estimates [33]. Estimating $\mathrm{p}_{\mathrm{ij}}$ was done using $\log \left(\mathrm{p}_{\mathrm{ij}} /\left(1-\mathrm{p}_{\mathrm{ij}}\right)\right)=\operatorname{sex}_{\mathrm{i}}+$ breed $_{\mathrm{j}}$, with $i$ being F, NF, M, NM, $j$ $=1,2, \ldots, 157$, sex $x_{\mathrm{i}}$ was the sex effect for group $i$ and breed $_{\mathrm{j}}$ was the impact of breed $j$ on disease prevalence. In our hierarchical Bayesian analysis we assumed the prior densities for the parameters of the model to be $\operatorname{sex}_{\mathrm{i}} \sim \mathrm{N}\left(0, \sigma_{s}^{2}\right)$ and breed $_{\mathrm{j}} \sim \mathrm{N}\left(0, \sigma_{b}^{2}\right)$. Because these conditions were relatively uncommon, and some of the breed groups relatively small, the weakly informative prior of a half-Cauchy for $\sigma_{s}^{2}$ and $\sigma_{b}^{2}$ in our hierarchical model can bring some valuable stability to our estimates of $\mathrm{p}_{\mathrm{ij}}$ and the subsequent OR values [34]. Specifically, we assume that $\sigma_{s}^{2} \sim$ Cauchy $(0,5)$ and $\sigma_{b}^{2} \sim$ Cauchy $(0,5)$, with both being limited to positive values. Evaluation of this hierarchical model was made possible with a Monte Carlo Markov Chain (MCMC) sampling process run in four chains. Each chain was based upon 20,000 total samples with the first 5,000 discarded as part of the warm-up process and the remainder thinned to every 20-th sample, resulting in a MCMC sample of 3,000 values [31]. The convergence to the posterior density was evaluated by the Gelman-Rubin test statistic and values below 1.05 indicate the adequacy of the MCMC sampling process for the data evaluated [35].

Having included breed in our analytic model, we were also able to evaluate the OR across the sex classes within each identified breed group. Accordingly, the ratios for males and females within breed group $j$ are $\mathrm{OR}_{\mathrm{Fj}}=\left[\mathrm{p}_{\mathrm{NF}} /\right.$
$\left.\left(1-\mathrm{p}_{\mathrm{NF}}\right)\right] /\left[\mathrm{p}_{\mathrm{Fj}} /\left(1-\mathrm{p}_{\mathrm{Fj}}\right)\right]$ and $\mathrm{OR}_{\mathrm{Mj}}=\left[\mathrm{p}_{\mathrm{NMj}} /\left(1-\mathrm{p}_{\mathrm{NMj}}\right)\right] /\left[\mathrm{p}_{\mathrm{Mj}} /\right.$ $\left.\left(1-\mathrm{p}_{\mathrm{Mi}}\right)\right]$, respectively, for all groups $j=1,2, \ldots, 157$. Like any OR, values that differ from 1.0 are considered evidence of inequality of disease risk across population subgroups. To evaluate the risk of neutering on disease prevalence we took advantage of the MCMC sampling process to compute the posterior probability of the OR exceeding 2.0. Choosing 2.0 rather than 1.0 permits a more cautious evaluation of the increase in disease risk that may be incurred from neutering. Being a probability, this value is bounded by the interval $[0,1]$ and thus can be clearly represented in a heat map to identify trends in disease risk across breed groups.

To evaluate the risk of neutering on disease prevalence within a breed or group we took advantage of the MCMC sampling process to compute the posterior probability of the OR exceeding 1.0. Being a probability, this value is bounded by the interval $[0,1]$ and thus can be clearly represented in a heat map to identify trends in disease risk across breed groups. To provide visual clarity to the heat map of disease prevalence, we divided the within breed posterior probabilities into one of five groups. Although a posterior probability is not equivalent directly to a hypothesis test, we consider here a general correspondence. That is, a posterior probability less than 0.05 implies that $95 \%$ of the time, the computed OR for a given within breed neuter status comparison was less than 1.0. Such an outcome is a strong indication that neutering significantly reduces the prevalence of disease. Similarly, a posterior probability greater than 0.95 implies that less than $5 \%$ of the computed OR values were less than 1 , providing strong evidence that neutering increases the prevalence of disease in this breed group. Expanding upon this simple interpretation of the posterior probability of an OR $>1$ we classified breeds groups, within a disease, into one of five groups: I. Posterior probability less than 0.05 , strong indication that neutering reduces disease prevalence; II. Posterior probability between 0.05 and 0.10 , evidence suggesting that neutering can reduce disease prevalence; III. Posterior probability between 0.10 and 0.90 , no convincing evidence that neutering impacts disease prevalence; IV. Posterior probability between 0.90 and 0.95 , evidence suggesting that neutering can increase disease prevalence; and V. Posterior probability greater than 0.95 , strong indication that neutering increases disease prevalence.

\section{Results}

In the study population of 90,090 dogs, 9,133 were $\mathrm{F}$, 36,574 were NF, 12,555 were $M$, and 31,838 were NM. All the conditions assessed were less than $5 \%$ of the overall dog population studied with the exception of IVDD which was diagnosed in nearly $6 \%$ of the dogs seen at the hospital (Table 1). Ventricular septal defect was the lowest at $0.17 \%$. Male and female condition 


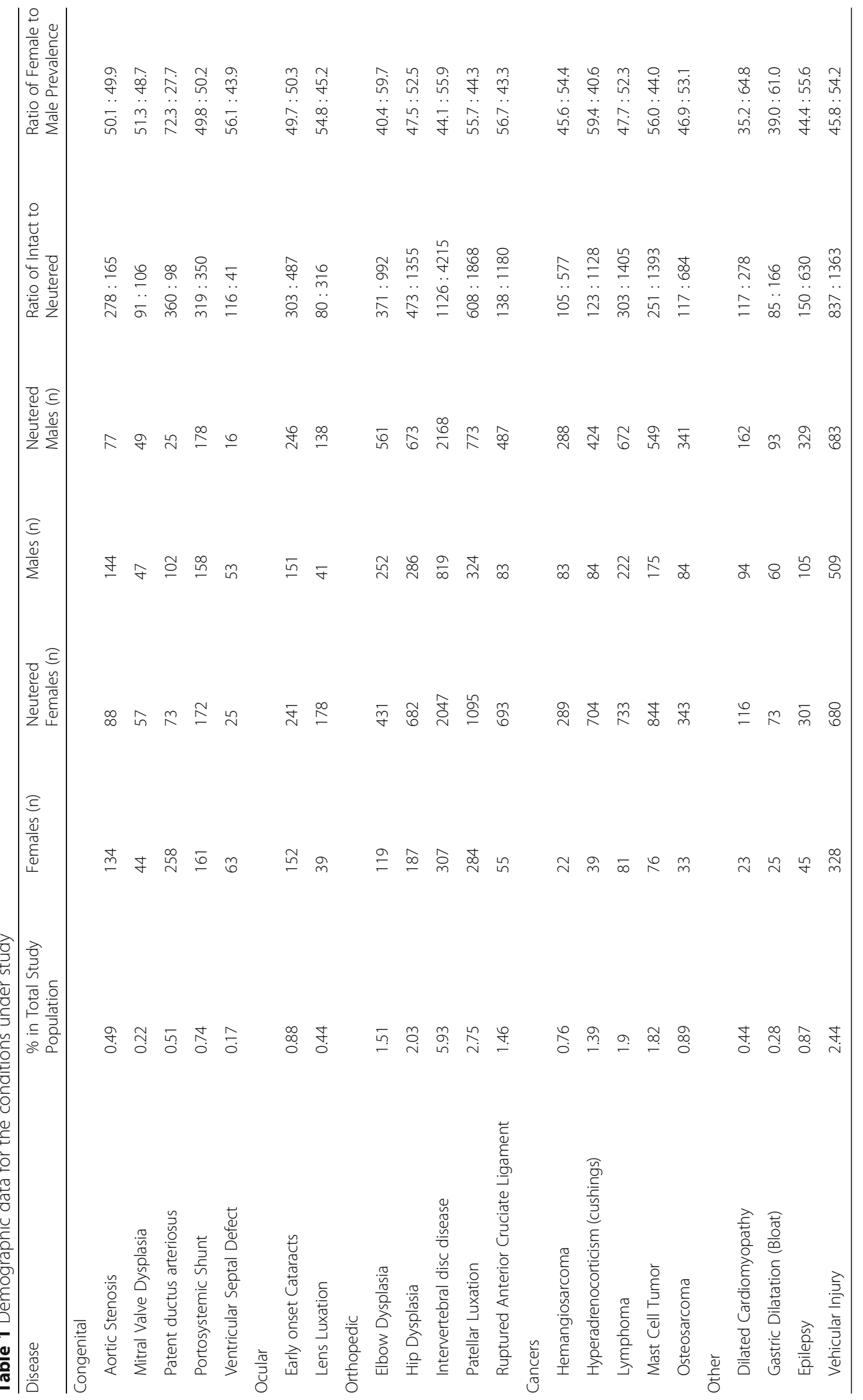


distribution, without consideration of neuter status, was $\sim 50 \%$ overall with some conditions being disproportionately higher in one sex vs. the other: PDA was higher in females and dilated cardiomyopathy and GDV higher in males (Table 1). Congenital conditions had the highest proportion recorded for intact dogs and for aortic stenosis, PDA, and ventricular septal defect in particular, intact dogs accounted for more than $60 \%$ of the cases.

The median age of neuter in the population for which age at neuter was known $(n=6,281)$ was 13.4 and 13.1 months for males and females, respectively [18]. The median age of neutering associated with early onset or congenital diseases was $7.54 \pm 1.56$ months for both males and females based upon known neuter ages of 262 dogs with these conditions (Fig. 1). For later onset inherited conditions, median age of neuter differed $(p<0.05)$ with females at 16.2 months $(n=371)$ and males at 22.8 months $(n=461)$. Intact dogs of both sexes tended to be diagnosed at earlier ages than neutered dogs for the majority of disorders (Fig. 2). Hyperadrenocorticism was the sole condition in which intact females and neutered females were diagnosed at statistically equivalent ages $(\mathrm{P}>0.05)$. For males, dilated cardiomyopathy, GDV, lens luxation, RACL, hyperadrenocorticism, hemangiosarcoma, lymphoma, mast cell tumor, and osteosarcoma were diagnosed at ages that did not statistically differ for intact and neutered dogs.

The risk of all congenital conditions assessed was greater for the intact dogs of both sexes as seen in Table 2 . Intact females were at 2.86 to 14 times higher risk than neutered females and intact males were at 2.3 to 10 times higher risk than neutered males for these early onset conditions. For the other conditions, intact dogs were at greater risk ( $\sim 50 \%$ increased risk) for early onset cataracts and being involved in vehicular injuries. Intact males were at greater risk ( 40-50\% increased risk) for dilated cardiomyopathy and GDV and neuter status was not associated with lens luxation, elbow or hip dysplasia, IVDD, or patellar luxation. For females, there was no significant association of neuter status with disease expression for dilated cardiomyopathy, GDV, lens luxation, elbow or hip dysplasia, or patellar luxation and neutering was associated with a 70\% increased risk of IVDD. For the cancers, epilepsy, and RACL, neuter status was positively associated with disease risk; males exhibited less of a risk associated with neutering than females.

The impacts of neutering within breeds was assessed, although extreme caution must be applied due to the small number of cases for many of the breeds (Additional file 1: Table S1). Nevertheless, inspecting the risk associated with neutering by breed may be informative (Additional file 2: Figures S1 and S2). The heat maps of risk, presented by AKC groups, reinforced the findings of the overall population, that is, when individual breeds were not considered. Exceptions in the overall population trend were seen in some breeds that showed either a reduction or enhancement of risk with neutering that differed from the aggregate risk, but upon closer inspection, the data were generally sparse for breeds showing a deviation from the overall trend. For example, osteosarcoma in breeds that showed reduced risk with neutering (Neopolitan mastiff, Airedale terrier, Bichon Frise, Basset hound, Bloodhound, Afghan hound, and Borzoi) all had zero cases in the NM sex class and one case in the M sex class which would correspond to a reduced risk with neutering. In conditions where there was no detected risk in the overall population certain breeds showed a reduced risk while others exhibited a

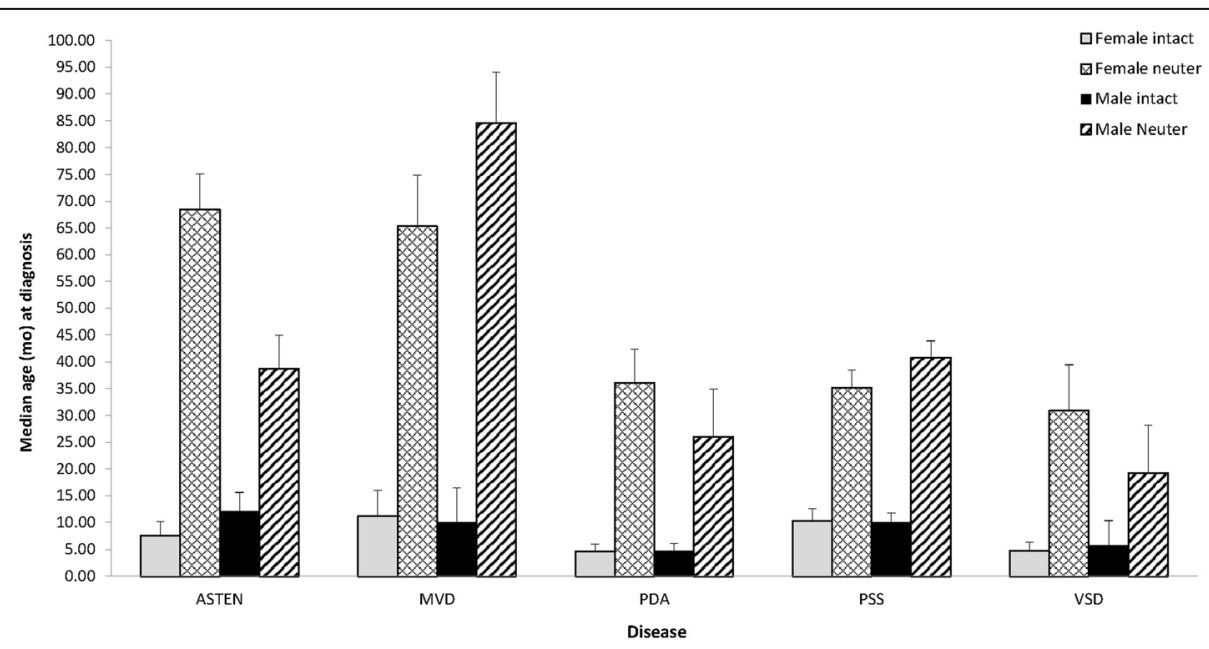

Fig. 1 Median age at reported diagnosis for the congenital conditions of aortic stenosis (ASTEN), mitral valve disease (MVD), patent ductus arteriosus (PDA), portosystemic shunt (PSS), and ventricular septal defect (VSD). Female intact are light gray bars, neutered female are light gray hatched bars, male intact are solid black bars, neutered males dark black hatched bars 

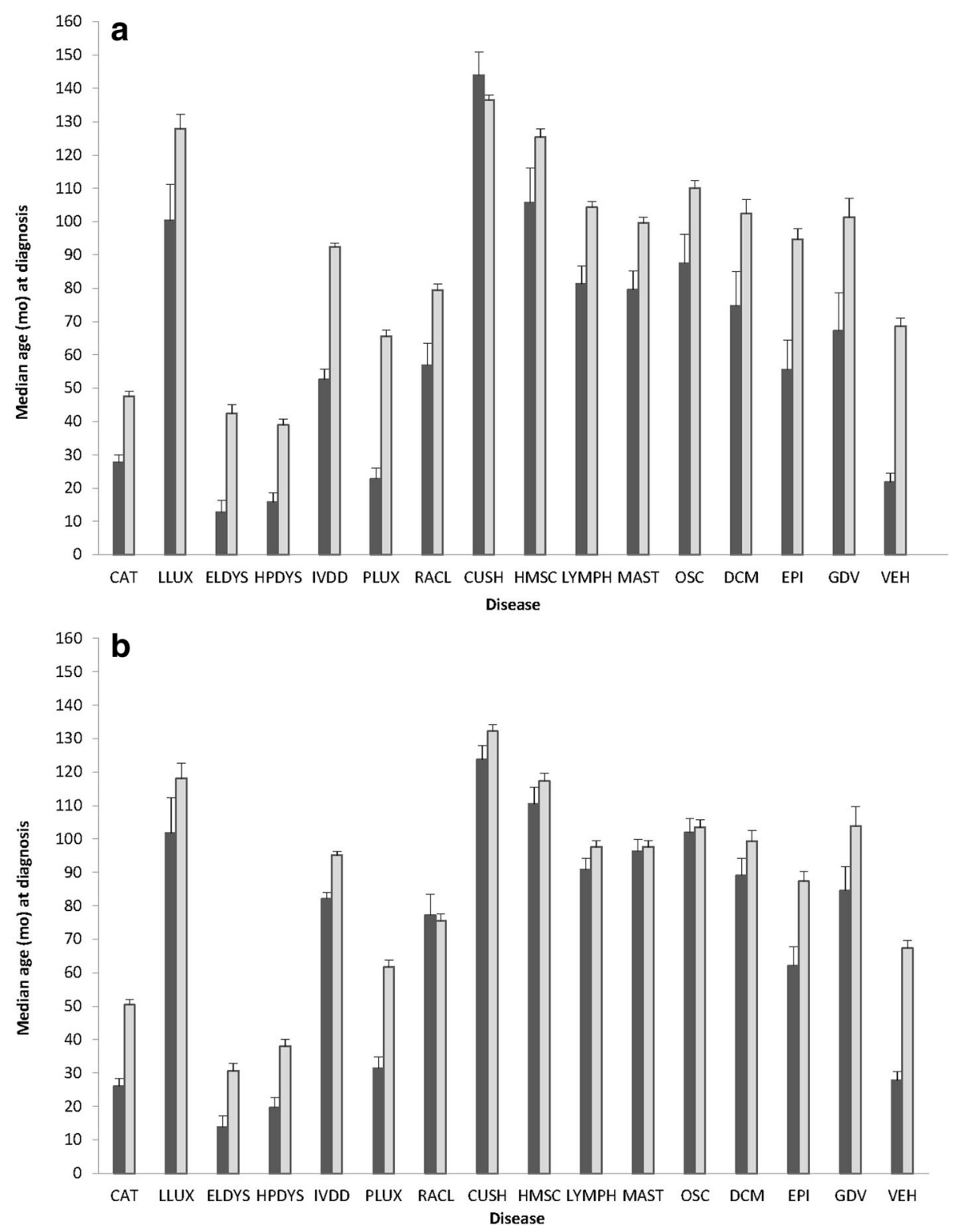

Fig. 2 Median age (and standard error) at reported diagnosis for genetic conditions and vehicular injury for intact (dark bars) and neutered (light bars) females (a) and males (b). Conditions assessed were cataracts (CAT), lens luxation (LLUX), elbow dysplasia (ELDYS), hip dysplasia (HPDYS), intervertebral disk disease (IVDD), patellar luxation (PLUX), ruptured anterior cruciate ligament (RACL), hyperadrenocorticism (CUSH), hemangiosarcoma (HMSC), lymphoma (LYMPH), mast cell tumor (MAST), osteosarcoma (OSC), dilated cardiomyopathy (DCM), epilepsy (EPI), gastric dilatation volvulus (GDV), and vehicular injury (VEH)

greater risk. For example, the associated neuter risk with hip and elbow dysplasia varied noticeably across breeds. Females and/or males of the Belgian Malinois, Bouvier des Flandres, Collie, Old English sheepdog, Puli, and Shetland sheepdog herding breeds presented different hip dysplasia responses from the aggregate population. Yet those breeds each had a sex class with zero cases. In contrast, German shepherd dogs with large numbers of cases in each sex class showed no association between neutering and risk for hip dysplasia consistent with the aggregate population.

The category of mixed breed presented an interesting scenario. For the congenital, IVDD, RACL, GDV, cancers, and vehicular injury conditions females and males of the mixed breed category followed the risk associations seen for the population as a whole. For elbow dysplasia, hip dysplasia, patellar luxation, DCM, early onset cataracts, and lens luxation there were differences in risk for the 
Table 2 The odds ratio (OR) ( \pm standard error) for the neutered dog relative to the intact dog for the conditions under study

\begin{tabular}{|c|c|c|}
\hline \multirow[b]{2}{*}{ Disease } & \multicolumn{2}{|c|}{ OR } \\
\hline & $\begin{array}{l}\text { Neutered } \\
\text { Female }\end{array}$ & $\begin{array}{c}\text { Neutered } \\
\text { Male }\end{array}$ \\
\hline \multicolumn{3}{|l|}{ Ocular } \\
\hline Early onset Cataracts & $0.42+0.04$ & $0.66+0.07$ \\
\hline Lens luxation & $1.13 \pm 0.17$ & $1.19 \pm 0.20$ \\
\hline \multicolumn{3}{|l|}{ Orthopedic } \\
\hline Elbow dysplasia & $0.91 \pm 0.09$ & $0.89 \pm 0.07$ \\
\hline Hip Dysplasia & $0.93 \pm 0.07$ & $0.96 \pm 0.06$ \\
\hline Intervertebral disk disease & $1.70+0.10$ & $1.06 \pm 0.04$ \\
\hline Patellar luxation & $0.99 \pm 0.06$ & $0.95 \pm 0.06$ \\
\hline Ruptured Anterior Cruciate Ligament & $3.18+0.45$ & $2.32+0.28$ \\
\hline \multicolumn{3}{|l|}{ Cancer } \\
\hline Hemangiosarcoma & $3.18+0.73$ & $1.39+0.17$ \\
\hline Hyperadrenocorticism (cushings) & $4.56+0.76$ & $2.02+0.24$ \\
\hline Lymphoma & $2.25+0.28$ & $1.20+0.09$ \\
\hline Mast cell tumor & $2.78+0.33$ & $1.25+0.11$ \\
\hline Osteosarcoma & $2.53+0.47$ & $1.62+0.20$ \\
\hline \multicolumn{3}{|l|}{ Other } \\
\hline Dilated Cardiomyopathy & $1.25 \pm 0.28$ & $0.70+0.09$ \\
\hline Gastric Dilatation Volvulus & $0.76 \pm 0.17$ & $0.65+0.11$ \\
\hline Epilepsy & $1.60+0.26$ & $1.25+0.14$ \\
\hline Vehicular Injury & $0.52+0.04$ & $0.53+0.03$ \\
\hline \multirow[t]{2}{*}{ Congenital Conditions } & \multicolumn{2}{|c|}{ OR } \\
\hline & $\begin{array}{l}\text { Neutered } \\
\text { Female }\end{array}$ & $\begin{array}{c}\text { Neutered } \\
\text { Male }\end{array}$ \\
\hline Aortic Stenosis & $0.17+0.02$ & $0.21+0.03$ \\
\hline Mitral valve disease & $0.35+0.07$ & $0.43+0.09$ \\
\hline Patent Ductus Arteriosus & $0.07+0.01$ & $0.10+0.02$ \\
\hline Portosystemic shunt & $0.27+0.03$ & $0.45+0.05$ \\
\hline Ventricular septal defect & $0.10+0.02$ & $0.13+0.04$ \\
\hline
\end{tabular}

Yellow shading indicates neutering was associated with reduced risk and dark gray shading indicates neutering was associated with an increased risk ( $p<0.05)$. No shading indicates no difference in risk

mixed breeds from that seen in the general population that represented either an increase in risk for neutered females (elbow and hip dysplasia, DCM, and cataracts) or a decrease risk in neutered males (patellar luxation). Neutered mixed breed females were at less risk for lens luxation than that seen for the general population.

\section{Discussion}

Numerous recent reports have centered on the advisability of neutering and unintended consequences associated with that surgical procedure $[12-14,19,36,37]$. In this study, the goal was to assess the association of gonadal hormones on the expression of various conditions presumed to be inherited by assessing risk of expression associated with neutering. Knowledge of the interplay of neutering with certain conditions may inform medical decisions related to neutering. The findings of the present work, a retrospective association study, are consistent with some conclusions drawn in published literature. Specifically, our results indicate that for some 
inherited conditions, neutering provides a benefit and is associated with reduced risk whereas for other conditions the risk was elevated in the neutered dog, most especially the cancers and RACL.

As for any retrospective study, one must be mindful of the limitations presented in the data. The hospital whose records were interrogated, serves as a referral hospital in addition to being the primary care clinic for a limited geographical region. The data therefore may be biased toward a subset of dogs and may not reflect the dog population risk for disease associated with neutering. The congruence of the risk found in the present study with risk reported in past studies would suggest that this bias is not prohibitive. The absence of recorded age of neuter for the majority of the patients precluded associating a cause and effect premise between neuter and disease expression or inclusion of weight changes following the neuter. A prospective study of enormous proportions comprised of numerous breeds and sufficient numbers of each sex class would be necessary to confirm the risks identified in the present study. However, without that type of prospective study, defining the cause and effect of neutering on the expression of all the conditions outlined here is impossible and therefore one must rely upon associations and retrospective analyses and the recognition that association is not a demonstration of causality.

For all the conditions assessed, the congenital conditions of aortic stenosis, PDA, and ventral septal defect were the only conditions in which intact dogs exhibited a greater prevalence than neutered dogs. Although not significantly so, mitral valve dysplasia and portosystemic shunt had relatively high prevalence in intact dogs compared to the prevalence seen for all other conditions. Congenital conditions affect a dog's quality of life and seemed to be diagnosed at relatively early ages with the dogs neutered soon thereafter likely accounting for the higher risk in intact dogs.

An increased risk associated with neutering for orthopedic conditions and cancers has reported previously for specific breeds [12-14]. For orthopedic conditions, typically the association is seen when early to late neutering is compared $[12,13,16]$ with a predisposition of neutered dogs to exhibit joint disorders, especially in dogs neutered before sexual maturity $[12,13,19,20]$. The association may be lost if the age of neuter is not accounted for as seen in the present study or the risk may be isolated to different breeds. A recent retrospective study by Hart et al. [14] highlighted the impact of breed on the risk associated with neutering in the expression of joint disorders; our breed data mirrored their findings that targeted Labrador and Golden retrievers. Terrier breeds tended to a greater risk with neutering whereas for nonsporting breeds, neutering was associated with a reduced risk. It is well understood that past sire and dam selection decisions within a breed alters gene frequency; disease expression patterns will be influenced by the interplay of altered gonadal hormones and the genetic background of the dogs [38, 39]. It must again be emphasized that low sample numbers of cases in each sex class requires caution in interpretation for individual breeds. The absence of significant risk observed for hip and elbow dysplasia and patellar luxation as reported in the present study for the aggregate dog population reflects that variable response in different breeds and in the variable statistical power based upon the number of cases for breeds.

In contrast to the above orthopedic conditions, cruciate ligament rupture is reported as a generalized risk in all neutered dogs $[16,40]$ a finding supported by the present study in which neutering of both sexes was associated with increased risk of RACL across breeds. The evidence for a protective role of sex hormones in ligament integrity can be seen in the presence of gonadal hormone receptors on ligaments [41], the increase of ligament elastin content and fiber diameter in response to estrogen administration [42], and the greater likelihood of cruciate injury in female athletes when estrogen is low [43]. However, obese dogs are four times more likely to rupture their cranial cruciate ligament [44] and neutering is a factor highly associated with the development of obesity, with neutered females being more prone than neutered males [45]. An obesity association, distinct from the gonadal hormone role, driving the observed increase in RACL cannot be discounted.

Of the genetic conditions evaluated in this study, IVDD was the most prevalent, a finding consistent with a previous retrospective study [46]. Previous studies also report that intact males have a one to two-fold higher IVDD prevalence than intact females [46-48]. As reviewed by Brisson [49], some studies report males and neutered females are at higher risk for IVDD. The elevated risk of IVDD in neutered females is also consistent with a recent study in rats that demonstrated ossification of the intervertebral disk endplate, which provides nutrients to the disk through marrow contact channels, in response to ovariectomy [50]. Upon estrogen supplementation of the ovariectomized animals, the authors observed an increase in channel volume, improved nutrient flow to the disk, and reduced IVDD progression. Similar impairment of vertebral bone structure is observed in post-menopausal women. As estrogen levels decline with menopause, bone structure is compromised, notably with intervertebral disk damage (as reviewed by [51-53]). Removing the positive effects of estrogen on bone by neutering female dogs would be expected to increase their risk for IVDD compared to intact females.

A presumed protective role of estrogen was seen in many of the assessed conditions, notably cancer. Cancer 
risk has been associated with neuter status in the literature $[11,54,55]$. In the present study, age of diagnosis for neutered and intact males was statistically equivalent for most cancers with the exception of lymphoma. In contrast, age of cancer diagnosis was statistically later for neutered females for all cancers except hyperadrenocorticism. This sex discrepancy of age at diagnosis may account for the greater risk observed for neutered females than neutered males. A greater risk for cancers associated with neutering may reflect the (non-significant) tendency for neutered dogs to be older when diagnosed and the greater longevity in neutered dogs $[11,56,57]$ as advancing age is associated with a greater prevalence of cancers $[24,58]$.

Importantly, however, is that not all cancers in dogs show an increased risk with neutering. The incidence of mammary cancer is virtually eliminated if females are neutered prior to sexual maturity [59] whereas the frequency of other cancers, such as melanoma and squamous cell carcinoma, are not influenced by neuter status [11]. What accounts for the susceptibility to cancer upon removal of gonadal steroids is unclear. An additional confounding effect is the role of neutering on the development of obesity and the association with joint disorders and increased incidence of cancers with obesity $[60,61]$. The elevated risk of the conditions seen in the present study, including the cancers, may be a secondary effect of increased body weight and altered metabolism [62, 63].

Interestingly, the risk of epilepsy was higher in the neutered population, coinciding with a report on dogs in the United Kingdom [64]. Gonadal hormones, most especially estrogen, has been shown to reduce the seizure threshold cells in both humans and laboratory animals whereas progesterone is considered to be "anticonvulsant" (reviewed in $[65,66]$. Despite those generalizations, estrogen has differential effects in animal models of epilepsy with it being promotional in some induced models and anticonvulsant in others [65]. Estrogens have also been suggested to be anticonvulsant when doses are physiological and not supraphysiological [67]. Progesterone on the other hand reduces the likelihood of seizures. The intact female dog has elevated progesterone for some months during its reproductive cycle which may account for a lowered risk for intact females. The role of testosterone in seizure response has been less well studied [67] and the risk seen in neutered males was relatively low compared to the intact males perhaps reflecting the conversion of endogenous testosterone to a protective estradiol form.

Retrospective studies of dogs have demonstrated breed predispositions in cataract prevalence with equivalent male female distribution, though neuter status was not recorded $[28,68]$. The cataracts analyzed in the present study displayed greater risk for intact dogs of both sexes with females showing a higher risk than males with a similar breed predisposition as reported in the above mentioned studies. The female sex hormones have been implicated in the development of lens opacities and cataracts [69] based upon observations that women develop cataracts more frequently than men, a greater cataract incidence occurs in post-menopausal women, and exogenous estrogen plays a protective role in ovariectomized rats [70]. However a study by Colitz et al., [71] showed that cataractous lens epithelial cells overexpress estrogen receptors and the authors posited that these cells may become estrogen sensitive during caractogenesis. Furthermore, lens epithelia have the aromatase pathway enabling conversion of testosterone to estradiol [72]. This would indicate a greater risk associated with intact dogs of both sexes.

The male preponderance for dilated cardiomyopathy [73] was seen in the present data with males accounting for $64.8 \%$ of the cases. Neutered males, but not females, had a reduced risk for dilated cardiomyopathy strongly suggesting a permissive effect of testosterone on expression of this disease, a supposition supported by the anabolic steroid literature. Administration of anabolic steroids to dogs results in hypertrophy of cardiac muscle [74] mimicking the cardiomyopathy conditions seen in anabolic steroid use in athletes [75].

The overall prevalence of GDV being greater in males than females agrees with published findings [76]. Although the present study indicates that neutering reduced the risk of GDV, significantly so in males, other published studies report variable impact of sex and neuter status ([77] and reviewed in [78]). The inconsistent association of neuter status on risk of GDV likely reflects the complex nature of the condition with the sex hormones being just one of the many factors that influence expression. Neutering was highly associated with increased risk within the breeds of the AKC working group; however, those breeds tended to have the highest prevalence of GDV.

The risk of vehicular injuries was also reduced in the neutered dogs possibly due to associated differences in owner behavior and/or dog behavior. A study on owner reported trainability indicated that for some breeds neutering improved trainability though the authors caution against broad generalization across breeds arguing more for breed specific assessment [79]. Dogs that have not been trained or do not respond to an owner's command may be more likely to enter traffic. Neutering has been associated with alterations in dog behavior, reducing the risk of biting in certain breeds of dogs [8] and neutered dogs are at reduced risk for relinquishment presumably due to improved behavior [10]. A study that evaluated boldness in dogs found that intact dogs of both sexes 
and multiple breeds are more "bold" than neutered dogs [80] which may be associated with exploratory and wandering behavior. Additionally, neutered dogs are less aggressive and less likely to roam [6,7]. These studies suggest that the impact of neutering on dog behavior could account for the reduced risk of vehicular injury.

Despite the limited ability to assess individual breed contribution to the risk associated with neutering, several key points can be drawn. The breeds making up the herding, non-sporting, sporting, and working AKC groups were more likely to display an association with neutering for risk of certain conditions. Also, the mixed breed group, which reflects an amalgamation of various breeds, would be expected to reflect the overall population risk response, which was for the most part observed, validating the approach of determining an overall dog population response. Finally, neutering in females presented a generally greater risk for the conditions than that detected in males.

\section{Conclusions}

Risk assessment based upon retrospective studies should be applied to clinical settings and client recommendations with caution because of the limitations of such studies. In the present study, factors such as age of neuter, breed, and concomitant metabolic conditions such as obesity were not incorporated into the analyses. Yet the fact of a neutering risk that exists for certain conditions, most especially in females, cannot be ignored and underscores the need for reflective consultation between the client and the clinician when considering neutering. For dogs not to be used in planned breedings, the convenience and advantages of neutering dogs must be weighed against possible risks associated with neutering.

\section{Additional files}

\section{Additional file 1: Table S1. Number of cases and controls for each} condition by breed used in the study. (XLSX $48 \mathrm{~kb}$ )

Additional file 2: Figures $\mathbf{S 1}$ and S2. Heat map of risk associated with neutering in females (Figure S1) and males (Figure S2) by dog breed, assembled into AKC breed groupings. Heat map represents classification of one of five categories: I. Posterior probability less than 0.05 , strong indication that neutering reduces disease prevalence (green); II. Posterior probability between 0.05 and 0.10 , evidence suggesting that neutering can reduce disease prevalence (light green/teal); III. Posterior probability between 0.10 and 0.90 , no convincing evidence that neutering impacts disease prevalence (blue); IV. Posterior probability between 0.90 and 0.95 , evidence suggesting that neutering can increase disease prevalence (peach); and V. Posterior probability greater than 0.95 , strong indication that neutering increases disease prevalence (red). Refer to Additional file 1 : Table S1 for breed names associated with breed codes. (ZIP $7915 \mathrm{~kb}$ )

\section{Abbreviations}

AKC: American Kennel Club; DCM: Dilated cardiomyopathy; F: Intact Female; FSS: Foundation Stock Service; GDV: Gastric dilatation volvulus:

IVDD: Intervertebral disk disease; M: Intact male; NF: Neutered female;
NM: Neutered Male; OR: Odds Ratio; PDA: Patent ductus arteriosus; RACL: Ruptured anterior cruciate ligament

\section{Acknowledgements}

We gratefully acknowledge the infrastructure support of the Department of Animal Science, College of Agricultural and Environmental Sciences, and the California Agricultural Experiment Station of the University of California-Davis as well as the University of California, Davis School of Veterinary Medicine and the veterinarians, staff, and students who diagnosed and cared for the patients.

\section{Funding}

Research was supported in part by W.K. Kellogg Endowment

\section{Availability of data and materials}

The dataset supporting the conclusions of this article is presented within the main manuscript.

\section{Authors' contributions}

JMB: data extraction and collation, interpretation, and manuscript preparation. TPB: data extraction and collation. DLB: input on data collection and manuscript presentation. TRF: informed data collection, determined and executed optimal design analysis, and edited the manuscript. AMO: project conception, design, analysis, and manuscript preparation. All authors read and approved the final manuscript.

\section{Competing interests}

The authors declare that they have no competing interests.

\section{Consent for publication}

Not applicable.

Ethics approval and consent to participate

The retrospective analysis is exempt from ethics approval from the institutional animal care and use committee.

\section{Publisher's Note}

Springer Nature remains neutral with regard to jurisdictional claims in published maps and institutional affiliations.

\section{Author details}

${ }^{1}$ Department of Animal Science, University of California, One Shields Ave, Davis, CA 95616, USA. ²Department of Population Health \& Reproduction, School of Veterinary Medicine, University of California, Davis, CA 95616, USA.

Received: 4 January 2017 Accepted: 4 May 2017

Published online: 26 May 2017

\section{References}

1. Kustritz MVR. Pregnancy diagnosis and abnormalities of pregnancy in the dog. Theriogenology. 2005;64(3):755-65.

2. McKenzie B. Evaluating the benefits and risks of neutering dogs and cats. CAB Rev Perspective Agriculture Vet Sci Nutr Nat Res. 2010;5:1-18.

3. Bryan JN, Keeler MR, Henry CJ, Bryan ME, Hahn AW, Caldwell CW. A population study of neutering status as a risk factor for canine prostate cancer. Prostate. 2007:67(11):1174-81.

4. Sorenmo K, Goldschmidt M, Shofer F, Goldkamp C, Ferracone J. Immunohistochemical characterization of canine prostatic carcinoma and correlation with castration status and castration time. Vet Comp Oncol. 2003;1(1):48-56.

5. Bell F, Klausner J, Hayden D, Feeney D, Johnston S. Clinical and pathologic features of prostatic adenocarcinoma in sexually intact and castrated dogs: 31 cases (1970-1987). J Am Vet Med Assoc. 1991;199(11):1623.

6. Maarschalkerweerd R, Endenburg N, Kirpensteijn J, Knol B. Influence of orchiectomy on canine behaviour. Vet Rec. 1997;140:617-9.

7. Hopkins S, Schubert T, Hart B. Castration of adult male dogs: effects on roaming, aggression, urine marking, and mounting. J Am Vet Med Assoc. 1976;168(12):1108-10.

8. Gershman KA, Sacks JJ, Wright JC. Which dogs bite? A case-control study of risk factors. Pediatrics. 1994;93(6):913-7. 
9. Casey RA, Loftus B, Bolster C, Richards GJ, Blackwell EJ. Human directed aggression in domestic dogs (Canis familiaris): occurrence in different contexts and risk factors. Appl Anim Behav Sci. 2014;152:52-63.

10. New Jr JC, Salman M, King M, Scarlett JM, Kass PH, Hutchison JM. Characteristics of shelter-relinquished animals and their owners compared with animals and their owners in US pet-owning households. J Appl Anim Welf Sci. 2000;3(3):179-201.

11. Hoffman JM, Creevy KE, Promislow DE. Reproductive capability is associated with lifespan and cause of death in companion dogs. PLoS ONE. 2013;8(4), e61082.

12. de la Riva GT, Hart BL, Farver TB, Oberbauer AM, Messam LLM, Willits N, Hart LA. Neutering dogs: effects on joint disorders and cancers in golden retrievers. PLOS ONE. 2013:8(2), e55937.

13. Hart BL, Hart LA, Thigpen AP, Willits NH. Long-term health effects of neutering dogs: comparison of labrador retrievers with golden retrievers. PLOS ONE. 2014;9(7):e102241.

14. Hart BL, Hart LA, Thigpen AP, Willits NH. Neutering of German shepherd dogs: associated joint disorders, cancers and urinary incontinence. Vet Med Sci. 2016;2:191-9.

15. Duerr FM, Duncan CG, Savicky RS, Park RD, Egger EL, Palmer RH. Risk factors for excessive tibial plateau angle in large-breed dogs with cranial cruciate ligament disease. J Am Vet Med Assoc. 2007;231(11):1688-91.

16. Witsberger TH, Villamil JA, Schultz LG, Hahn AW, Cook JL. Prevalence of and risk factors for hip dysplasia and cranial cruciate ligament deficiency in dogs. J Am Vet Med Assoc. 2008;232(12):1818-24.

17. Hart BL. Effect of gonadectomy on subsequent development of age-related cognitive impairment in dogs. J Am Vet Med Assoc. 2001;219(1):51-6.

18. Sundburg CR, Belanger JM, Bannasch DL, Famula TR, Oberbauer AM Gonadectomy effects on the risk of immune disorders in the dog: a retrospective study. BMC Vet Res. 2016;12(1):278.

19. Kustritz R. Effects of surgical sterilization on canine and feline health and on society. Reprod Domest Anim. 2012;47(s4):214-22.

20. Kustritz MVR. Determining the optimal age for gonadectomy of dogs and cats. J Am Vet Med Assoc. 2007;231(11):1665-75.

21. Smith AN. The role of neutering in cancer development. Vet Clin N Am Small Anim Pract. 2014:44(5):965-75.

22. Bellumori TP, Famula TR, Bannasch DL, Belanger JM, Oberbauer AM. Prevalence of inherited disorders among mixed-breed and purebred dogs: 27,254 cases (1995-2010). J Am Vet Med Assoc. 2013;242(11):1549-55.

23. Genevois J-P, Remy D, Viguier E, Carozzo C, Collard F, Cachon T, Maitre P, Fau D. Prevalence of hip dysplasia according to official radiographic screening, among 31 breeds of dogs in France-A retrospective study. Vet Comp Orthop Traumatol. 2008;21(1):21-4

24. Bronson R. Variation in age at death of dogs of different sexes and breeds. Am J Vet Res. 1982:43(11):2057-9.

25. Martin RA. Congenital portosystemic shunts in the dog and cat. Vet Clin N Am Small Anim Pract. 1993;23(3):609-23.

26. Tobias KM, Rohrbach BW. Association of breed with the diagnosis of congenital portosystemic shunts in dogs: 2,400 cases (1980-2002). J Am Vet Med Assoc. 2003;223(11):1636-9.

27. Tidholm A. Retrospective study of congenital heart defects in 151 dogs. J Small Anim Pract. 1997;38(3):94-8.

28. Gelatt KN, MacKay EO. Prevalence of primary breed-related cataracts in the dog in North America. Vet Ophthalmol. 2005;8(2):101-11.

29. Peterson ME. Diagnosis of hyperadrenocorticism in dogs. Clin Tech Small Anim Pract. 2007;22(1):2-11.

30. Gordis L. Epidemiology. 4th ed. Philadelphia: Saunders Elsevier; 2009

31. Carpenter B, Gelman A, Hoffman M, Lee D, Goodrich B, Betancourt M, Brubaker MA, Guo J, Li P, Riddell A. Stan: a probabilistic programming language. J Stat Softw. 2017;76(1):1-32.

32. R RDCT. R: A language and environment for statistical computing. In: R foundation for statistical computing. 2015.

33. Gelman A, Carlin JB, Stern HS, Rubin DB. Bayesian data analysis, vol. 2. Boca Raton: Taylor \& Francis; 2014

34. Gelman A. Prior distributions for variance parameters in hierarchical models (comment on article by Browne and Draper). Bayesian Anal. 2006;1(3):515-34.

35. Gelman A, Rubin D. Inference from iterative simulation using multiple sequences. Stat Sci. 1992;7(4):457-72.

36. Kustritz MVR. Early spay-neuter: clinical considerations. Clin Tech Small Anim Pract. 2002;17(3):124-8.
37. Kustritz MVR. Pros, cons, and techniques of pediatric neutering. Vet Clin N Am Small Anim Pract. 2014;44(2):221-33.

38. Oberbauer A, Belanger J, Bellumori T, Bannasch D, Famula T. Ten inherited disorders in purebred dogs by functional breed groupings. Canine Gene Epidemiol. 2015;2(1):1.

39. Pedersen NC, Brucker L, Tessier NG, Liu H, Penedo MCT, Hughes S, Oberbauer A, Sacks B. The effect of genetic bottlenecks and inbreeding on the incidence of two major autoimmune diseases in standard poodles, sebaceous adenitis and Addison's disease. Canine Gene Epidemiol. 2015;2(1):1

40. Slauterbeck J, Pankratz K, Xu K, Bozeman S, Hardy D. Canine ovariohysterectomy and orchiectomy increases the prevalence of $\mathrm{ACL}$ injury. Clin Orthop Relat Res. 2004;429:301-5.

41. Sciore P, Frank CB, Hart DA. Identification of sex hormone receptors in human and rabbit ligaments of the knee by reverse transcriptionpolymerase chain reaction: evidence that receptors are present in tissue from both male and female subjects. J Orthop Res. 1998;16(5):604-10.

42. Shikata J, Sanada H, Yamamuro T, Takeda T. Experimental studies of the elastic fiber of the capsular ligament: influence of ageing and sex hormones on the hip joint capsule of rats. Connect Tissue Res. 1979;7(1):21-7.

43. Slauterbeck JR, Fuzie SF, Smith MP, Clark RJ. The menstrual cycle, sex hormones, and anterior cruciate ligament injury/Commentary/Authors' response. J Athl Train. 2002;37(3):275.

44. Adams P, Bolus R, Middleton S, Moores A, Grierson J. Influence of signalment on developing cranial cruciate rupture in dogs in the UK. J Small Anim Pract. 2011;52(7):347-52.

45. Courcier E, Thomson R, Mellor D, Yam P. An epidemiological study of environmental factors associated with canine obesity. J Small Anim Pract. 2010;51(7):362-7.

46. Fluehmann $G$, Doherr $M$, Jaggy A. Canine neurological diseases in a referral hospital population between 1989 and 2000 in Switzerland. J Small Anim Pract. 2006:47(10):582-7.

47. Priester WA. Canine intervertebral disc disease-occurrence by age, breed, and sex among 8,117 cases. Theriogenology. 1976;6(2):293-303.

48. Itoh H, Hara Y, YOSHIMI N, Harada Y, Nezu Y, Yogo T, Ochi H, Hasegawa D, Orima $\mathrm{H}$, Tagawa M. A retrospective study of intervertebral disc herniation in dogs in Japan: 297 cases. J Vet Med Sci. 2008;70(7):701-6.

49. Brisson BA. Intervertebral disc disease in dogs. Vet Clin N Am Small Anim Pract. 2010;40(5):829-58

50. Jia H, Ma J, Lv J, Ma X, Xu W, Yang Y, Tian A, Wang Y, Sun L, Xu L. Oestrogen and parathyroid hormone alleviate lumbar intervertebral disc degeneration in ovariectomized rats and enhance Wnt/ $\beta$-catenin pathway activity. Scientific Reports. 2016;6:27521.

51. Wang Y-XJ, Griffith JF. Effect of menopause on lumbar disk degeneration: potential etiology 1. Radiology. 2010;257(2):318-20.

52. Lou C, Chen H, Feng X, Xiang G, Zhu S, Tian N, Jin Y, Fang M, Wang C, Xu $\mathrm{H}$. Menopause is associated with lumbar disc degeneration: a review of 4230 intervertebral discs. Climacteric. 2014;17(6):700-4.

53. Wei A, Shen B, Williams LA, Bhargav D, Yan F, Chong BH, Diwan AD. Expression and functional roles of estrogen receptor GPR30 in human intervertebral disc. J Steroid Biochem Mol Biol. 2016:158:46-55.

54. Zink MC, Farhoody P, Elser SE, Ruffini LD, Gibbons TA, Rieger RH. Evaluation of the risk and age of onset of cancer and behavioral disorders in gonadectomized Vizslas. J Am Vet Med Assoc. 2014;244(3):309-19.

55. Reichler I. Gonadectomy in cats and dogs: a review of risks and benefits. Reprod Domest Anim. 2009;44(s2):29-35.

56. Kraft W. Geriatrics in canine and feline internal medicine. Eur J Med Res. 1998:3(1-2):31-41.

57. Greer KA, Canterberry SC, Murphy KE. Statistical analysis regarding the effects of height and weight on life span of the domestic dog. Res Vet Sci. 2007:82(2):208-14.

58. Kelsey JL, Moore AS, Glickman LT. Epidemiologic studies of risk factors for cancer in pet dogs. Epidemiol Rev. 1998;20(2):204-17.

59. Schneider R, Dorn CR, Taylor D. Factors influencing canine mammary cancer development and postsurgical survival. J Natl Cancer Inst. 1969:43(6):1249-61.

60. German AJ. The growing problem of obesity in dogs and cats. J Nutr. 2006:136(7):1940S-6.

61. Lund EM, Armstrong PJ, Kirk CA, Klausner JS. Prevalence and risk factors for obesity in adult dogs from private US veterinary practices. Int J Appl Res Vet Med. 2006;4(2):177. 
62. McGreevy P, Thomson P, Pride C, Fawcett A, Grassi T, Jones B. Prevalence of obesity in dogs examined by Australian veterinary practices and the risk factors involved. Vet Rec English Ed. 2005;156(22):695-701.

63. Colliard L, Ancel J, Benet J-J, Paragon B-M, Blanchard G. Risk factors for obesity in dogs in France. J Nutr. 2006;136(7):1951S-4.

64. Short A, Dunne A, Lohi H, Boulton S, Carter S, Timofte D, Ollier W. Characteristics of epileptic episodes in UK dog breeds: an epidemiological approach. Vet Rec English Ed. 2011;169(2):48.

65. Scharfman HE, MacLusky NJ. The influence of gonadal hormones on neuronal excitability, seizures, and epilepsy in the female. Epilepsia. 2006:47(9):1423-40.

66. Woolley CS, Schwartzkroin PA. Hormonal effects on the brain. Epilepsia. 1998;39(s8):S2-8.

67. Van Meervenne SA, Volk HA, Matiasek K, Van Ham LM. The influence of sex hormones on seizures in dogs and humans. Vet J. 2014;201(1):15-20.

68. Donzel E, Arti L, Chahory S. Epidemiology and clinical presentation of canine cataracts in France: a retrospective study of 404 cases. Vet Ophthalmol. 2016.

69. Leibowitz HM, Krueger D, Maunder L, Milton R, Kini M, Kahn H, Nickerson R, Pool J, Colton T, Ganley J. The Framingham Eye Study monograph: an ophthalmological and epidemiological study of cataract, glaucoma, diabetic retinopathy, macular degeneration, and visual acuity in a general population of 2631 adults, 1973-1975. Surv Ophthalmol. 1979;24(Suppl):335-610.

70. Bigsby RM, Cardenas H, Caperell-Grant A, Grubbs CJ. Protective effects of estrogen in a rat model of age-related cataracts. Proc Natl Acad Sci. 1999;96(16):9328-32.

71. Colitz C, Chandler H, Lu P, Sugimoto Y, Barden C, Metzler A, Wilkie D, Bras I, Kuonen V, Robbin T. Estrogen Localization and Local Synthesis of Estrogen by Cataractous LEC. Invest Ophthalmol Vis Sci. 2005;46(13):2893.

72. Colitz CM, Lu P, Sugimoto Y, Barden CA, Chandler HL. Estradiol biosynthesis in canine lens epithelial cells. Curr Eye Res. 2015;40(5):541-8.

73. Tidholm A, Jönsson L. A retrospective study of canine dilated cardiomyopathy (189 cases). J Am Anim Hosp Assoc. 1996;33(6):544-50.

74. Rämö P. Anabolic steroids alter the haemodynamic responses of the canine left ventricle. Acta Physiol Scand. 1987;130(2):209-17.

75. Ferrera P, Putnam D, Verdile V. Anabolic steroid use as the possible precipitant of dilated cardiomyopathy. Cardiology. 1997;88(2):218-20.

76. Glickman LT, Glickman NW, Schellenberg DB, Raghavan M, Lee TL. Incidence of and breed-related risk factors for gastric dilatation-volvulus in dogs. J Am Vet Med Assoc. 2000;216(1):40-5.

77. Moore GE, Burkman KD, Carter MN, Peterson MR. Causes of death or reasons for euthanasia in military working dogs: 927 cases (1993-1996). J Am Vet Med Assoc. 2001;219(2):209-14.

78. Bell JS. Inherited and predisposing factors in the development of gastric dilatation volvulus in dogs. Top Companion Anim Med. 2014;29(3):60-3.

79. Serpell JA, Hsu YA. Effects of breed, sex, and neuter status on trainability in dogs. Anthrozoös. 2005;18(3):196-207.

80. Starling MJ, Branson N, Thomson PC, McGreevy PD. Age, sex and reproductive status affect boldness in dogs. Vet J. 2013;197(3):868-72

\section{Submit your next manuscript to BioMed Central and we will help you at every step:}

- We accept pre-submission inquiries

- Our selector tool helps you to find the most relevant journal

- We provide round the clock customer support

- Convenient online submission

- Thorough peer review

- Inclusion in PubMed and all major indexing services

- Maximum visibility for your research

Submit your manuscript at www.biomedcentral.com/submit 